\title{
Coming to a Consensus on Informed Consent for Case Reports
}

\author{
Mark Neavyn • Christine Murphy
}

Published online: 19 August 2014

(C) American College of Medical Toxicology 2014

\section{Background}

As social media streams unfiltered personal information, the boundaries of privacy are being redefined. Modern technology permits near-instant access to news and information. Easy and open access to information places medical journals in a particularly vulnerable position when it comes to publishing case reports. Case reports provide practitioners with precedent in the treatment of a rare or unique illness, and in medical toxicology, often represent the only published guidance on rare exposures. Internet search engines quickly link published reports on unique poisoning cases with social media and news outlet reports, allowing for a potential, and often inadvertent, violation of patient privacy rights. Medical journals, as with other forms of publication, are subject to legal scrutiny regarding negligent publishing and personal privacy rights, but more poignantly, authors and editors have an ethical responsibility to protect patient health information. How then do we balance our duty to protect patient identity with our responsibility to the Journal of Medical Toxicology (JMT) mission of "advancing the science and practice of medical toxicology"?

\footnotetext{
A Note from the Editor This commentary was developed by a working group of members of the JMT editorial board. The final version of this manuscript was reviewed and agreed upon by our editorial board.
}

M. Neavyn

Division Medical Toxicology, Department of Emergency Medicine, Hartford Hospital, Hartford, CT, USA

C. Murphy $(\square)$

Division of Medical Toxicology, Department of Emergency

Medicine, Carolinas Medical Center, Charlotte, NC, USA

e-mail: christine.murphy66@gmail.com

\section{Recent Precedents}

The World Medical Association has published an updated Declaration of Helsinki, which details the ethical responsibilities of researchers to protect human subjects [1]. Most institutional review boards, however, do not categorize case reports as human research. To address this chasm between human research and medical publishing ethics, an international consensus on the subject of patients' right to privacy in medical publications was reached in 1995 [2]. The Vancouver Group, made up of editors from 11 prominent international medical journals, determined that informed consent is a prerequisite to any written description of a patient that cannot be completely anonymized and that no identifiable information can be published. In parallel with the Declaration of Helsinki, a primary concern voiced by the Vancouver Group was the protection of vulnerable patients, such as patients who are comatose or with altered levels of consciousness. In such cases, it recommended informed consent be obtained from a legally authorized representative or surrogate. In addition, the Vancouver group stated, "complete anonymity is difficult to achieve, and informed consent should be obtained if there is any doubt" [2]. The position statement by the Vancouver Group suggested this requirement be included in a journal's instructions for authors. The degree of acceptable case subject anonymity remains a subject of debate for many medical journals.

Medical journals have a varied approach to the subject of informed consent for case reports, and few provide a definition of "identifiable information." For example, dermatologists communicate many of their clinical pearls through imagery. In JAMA Dermatology, anonymity is determined by the patient's ability to identify oneself in a photo, and any photo containing recognizable features requires informed consent. The New England Journal of Medicine has no defined informed consent for case reports, but a consent form exists 
for "identifiable patients." $B M J$ states in its instructions for authors, "publication of any personal information about an identifiable living patient requires the explicit consent of the patient or guardian." The Lancet requires consent "where you wish to include any case details, personal information, and/or images of patients or other individuals in order to comply with all applicable laws and regulations concerning privacy and/or security of personal information." JAMA requires informed consent for any patient "description" and requests a copy of the signed consent to publish. Annals of Emergency Medicine editor-in-chief, Michael Callaham, wrote an article describing the journal's policies on publication ethics, but he did not specifically address the question of consent for case reporting [3]. The Journal of Emergency Medicine only requires permission to publish patient photos and case details, but they do not specifically address consent to publish case reports. Clinical Toxicology requires that either patient consent for publication or an exemption statement from the authors' institutional review board should be submitted with the manuscript.

Publishing companies have similarly varied approaches to the question of informed consent for case reports. Elsevier presumes that ultimate responsibility lies with the reviewer and editor to determine confidentiality and anonymity [4]. Informa Healthcare provides a universal patient consent form for patient material (case history) or photos [5]. Finally, the publisher for this journal, Springer, states in their instructions for authors that "identifying details should be omitted if they are not essential." [5]. The publisher further echoes the Vancouver Group and states, "complete anonymity is difficult to achieve, however, and informed consent should be obtained if there is any doubt" [6].

\section{Consenting the Deceased}

Consenting the deceased has its own challenges and is a common problem encountered by the medical toxicologist. In the UK, personal health information is protected by the Data Protection Act of 1998 [7]. This Act does not protect the personal information of the deceased. In the US, the Health Insurance Portability and Accountability Act of 1996 (HIPAA) protects citizens' personal health information (PHI). This protection, unlike the Data Protection Act, is extended 50 years after the person's death [8]. This is only significant for a fraction of US-based case reports, as most authors do not include PHI identifiers as defined by HIPAA (see Table 1). However, in any case where a "unique identifying characteristic" is included, consent should be obtained. In some cases, the manner of poisoning/intoxication or the details surrounding a patient's death after poisoning/ intoxication may be the "unique identifying characteristic" and lead to inadvertent patient identification. BMJ suggests that authors attempt to consent the family of the deceased "as a
Table 1 HIPAA PHI identifiers [9]

PHI identifiers

1. Names

2. All geographical subdivisions smaller than a state

3. All elements of dates (except year) for dates directly related to an individual, including birth date, admission date, discharge date, date of death; and all ages over 89 and all elements of dates (including year) indicative of such age, except that such ages and elements may be aggregated into a single category of age 90 or older

4. Phone numbers

5. Fax numbers

6. Electronic mail addresses

7. Social Security numbers

8. Medical record numbers

9. Health plan beneficiary numbers

10. Account numbers

11. Certificate/license numbers

12. Vehicle identifiers and serial numbers, including license plate numbers

13. Device identifiers and serial numbers;

14. Web universal resource locators (URLs)

15. Internet protocol (IP) address numbers

16. Biometric identifiers, including finger and voice prints

17. Full face photographic images and any comparable images

18. Any other unique identifying number, characteristic, or code

matter of courtesy and medical ethics," and if consent is impossible the case must be completely anonymized. The journal further suggests that this may rarely require the authors to become anonymous as well. This approach seems reasonable for those case reports originating in the US that contain potentially "unique identifying characteristics" and involve deceased patients.

Authors, editors, and publishers face complex questions when deciding to publish personal health information, and there are circumstances where informed consent is difficult or impossible to obtain. The Committee on Publication Ethics (COPE) provides a forum for the anonymous discussion of this and other ethical dilemmas faced in medical publishing (http://publicationethics.org/). The challenge of consenting the deceased is one of many topics discussed in this forum. In one case from 2002, the editors of a journal describe a manuscript detailing four children who died of rare congenital heart anomalies. The cases included sufficient detail to identify the children; however, the author believed it was not possible to obtain consent years later from parents of a deceased child. COPE believed the editor had the authority to publish if the editor felt the benefit of disseminating the educational content outweighed the risk of revealing the identity of a case patient, but the editor would have to be prepared to defend such a stance. A pervasive theme from the COPE forum on this and other cases is that educational valor 
rarely outweighs patient confidentiality, regardless of the patient's mortal status. These conflicting guidelines make it extremely difficult to develop a position in this debate.

\section{Case Report Consent and Medical Toxicology}

There have been no position statements, reviews, or editorials by the medical toxicology community on the role of informed consent for case reports. Today, the JMT Editorial Board would request authors consider consent for all case reports, but will only require it at this time for cases including information that would allow for easy identification of the patient by an independent observer, such as cases of child or elder abuse or those under criminal investigation which are at high risk for discovery. Likewise, cases receiving media coverage will also be subject to public scrutiny. For cases where there is concern about adequate patient anonymity, the section editor may choose to submit the manuscript for peer-review to determine the need for consent through a consensus of reviewers. If a case appears to be clearly identifiable and consent is not provided, then the section editor will request consent prior to publication. This will be done at the discretion of the section editor and editor-in-chief. Most institutions have their own consent forms and at this time we will accept written attestation by the author in the manuscript stating consent to publish was obtained from the author(s) institution (Table 2).

High profile overdoses, poisonings, environmental exposures, and child abuse cases all depict vulnerable and frequently identifiable subjects and case reporting of these events may be easily linked to news reports. The JMT Editorial Board recognizes the perilous balance between the advancement in medical knowledge of the poisoned patient and protection of individual patient health information. It is a double-edged sword for medical toxicology, as information on rare and unique poisonings cannot be reproduced in original research and stems from case reports. Unfortunately, many of these case reports are inherently newsworthy. The editorial board of JMT agrees that obtaining consent on every case report is not always possible, and at this time feel this should not impede the dissemination of rare and/or unique poisoning cases. However, we recognize and support the push toward increased protection of patient privacy in scientific publishing, and feel it is important for authors to be aware of this trend.

We understand any move to require consent for all case reports is very controversial and there are many concerns about the effects this might have on the medical toxicology literature. However, we also feel it is important to maintain ethical responsibility in our writing and publishing. Given the enhanced accessibility to personal, identifiable information,
Table 2 Instructions for authors regarding consent for case reports

Instructions for authors

1. Attempt to obtain consent to publish for all case reports. Consent is only required for those case reports with highly identifiable patient information/characteristics included or cases of child abuse, elder abuse, and/or those involving criminal investigation. If consent is unobtainable (not refused) for one of these potentially identifiable cases, please make every effort to anonymize the case report and include a statement regarding the circumstances making consent unobtainable in a cover letter to the editor. The section editor and editor-in-chief will make decisions regarding these cases.

2. Consent forms from the primary author(s) institution are acceptable. Authors that do not have access to an institutional consent form should use the appropriate template provided by the World Health Organization found here: http://www.who.int/rpc/research_ethics/ informed_consent/en/

3. Authors are to obtain consent, provide a statement in the manuscript documenting patient consent obtained, and maintain copies of the signed consent form for a period of 7 years. In an effort to maintain patient privacy, copies of actual forms will only be requested for submission at the discretion of the editor-in-chief.

the editorial board at JMT feels this is an important discussion to acknowledge in an effort to ensure patient privacy and protect the integrity of our journal. As we continue to reevaluate our responsibilities to both our patients and the field of medical toxicology, we welcome your feedback and insight.

\section{References}

1. World Medical Association (2013) World Medical Association Declaration of Helsinki: ethical principles for medical research involving human subjects. JAMA 310(20):2191-4

2. International Committee of Medical Journal Ethics (1995) Protection of patients' rights to privacy. BMJ 311(7015):1272

3. Callaham ML (2003) Journal policy on ethics in scientific publication. Ann Emerg Med 41(1):82-9

4. Elsevier. Publishing Ethics. http://www.elsevier.com/about/ publishing-guidelines/publishing-ethics. Accessed 7 Apr 2014

5. Springer. Resources for Editors and Authors. http://informahealthcare. com/page/resources/authors. Accessed 7 April 2014

6. Springer. Statement of Informed Consent. http://www.springer.com/ authors?SGWID=0-111-6-608209-0. Accessed 7 April 2014

7. United Kingdom Parliament. Data Protection Act 1998. http://www. legislation.gov.uk/ukpga/1998/29/contents Accessed 7 April 2014

8. United States of America Office for Civil Rights. Health Information Privacy. http://www.hhs.gov/ocr/privacy/hipaa/faq/decedents/1500. html. Accessed 7 April 2014

9. United States Department of Health and Human Services. Health Information Privacy. http://www.hhs.gov/ocr/privacy/hipaa/ understanding/coveredentities/De-identification/guidance.html. Accessed 10 June 2014 